\title{
MEASUREMENT OF THE TRANSVERSE SPIN DEPENDENCE OF THE pp TOTAL CROSS SECTION IN THE 1-3 GeV/c REGION
}

\author{
Ed.K. BIEGERT, J.A. BUCHANAN, J.M. CLEMENT, W.H. DR AGOSET, R.D. FELDER ${ }^{1}$, \\ J.H. HOFTIEZER, K.R. HOGSTROM ${ }^{2}$, J. HUDOMALJ-GABITZSCH ${ }^{3}$, J.D. LESIKAR, \\ W.P. MADIGAN, G.S. MUTCHLER, G.C. PHILLIPS, J.B. ROBERTS and T.M. WILLIAMS ${ }^{4}$ \\ T.W. Bonner Nuclear Laboratories and Department of Physics, Rice University, Houston, Texas 77005, USA
}

K. ABE, R.C. FERNOW and T.A. MULERA ${ }^{5}$

Randall Laboratory of Physics, The University of Michigan, Ann Arbor, Michigan 48104, USA

and

S. BART, B.W. MAYES and L. PINSKY

Department of Physics, The University of Houston, Houston, Texas 77004, USA

Received 7 December 1977

The pp total cross section difference between pure transverse spin states was measured in the laboratory momentum range 1-3 GeV/c. Significant differences were found and these differences show striking energy dependence. This structure is in disagreement with the predictions of simple exchange models.

The difference in the pp total cross section for pure transverse spin states, $\Delta \sigma_{\mathrm{T}}=\sigma_{\mathrm{T}}(\uparrow \downarrow)-\sigma_{\mathrm{T}}(\uparrow \uparrow)$, is particularly simple in terms of spin amplitudes, since $\Delta \sigma_{\mathrm{T}}$ is proportional to $\operatorname{Im} \phi_{2}(t=0)$, where $\phi_{2}=\langle+|| \phi \mid-\rightarrow\rangle$ is the double flip, no net flip, s-channel helicity amplitude [1]. Similarly, the difference for pure initial longitudinal states, $\Delta \sigma_{\mathrm{I}}=\sigma_{\mathrm{T}}(\overrightarrow{\mathrm{Z}})-\sigma_{\mathrm{T}}(\rightrightarrows)$ is proportional to $\operatorname{Im}\left(\phi_{1}-\phi_{3}\right)$, where $\phi_{1}=\langle++|\phi|++\rangle$ and $\phi_{3}=$ $\langle+-|\phi|+-\rangle$ are the parallel and anti-parallel, non-flip, s-channel helicity amplitudes. Both of these crosssection differences might be expected to show striking structure as a function of energy as the thresholds for

Work supported by U.S. Energy Research and Development Administration.

1 Present address: Exxon Production Research Co., Houston, TX, USA.

2 Present address: Univ. N. Mex. School of Medicine, Albuquerque, NM, USA.

3 Present address: Teledyne Corporation, Houston, TX, USA.

${ }^{4}$ Present address: University of Houston, Houston, TX, USA.

5 Present address: Rice University, Houston, TX, USA. the production of various $\mathrm{N}^{*}$ 's are crossed [2-6]. Previous measurements of $\Delta \sigma_{\mathrm{T}}$ have indeed indicated a strong energy dependence in the range $2-3 \mathrm{GeV} / \mathrm{c}$ $[7,8]$. We have extended the measurement of $\Delta \sigma_{T}$ over the range $1-3 \mathrm{GeV} / c$.

The measurement was a standard "good geometry" attenuation experiment laid out as shown in fig. 1. The incident polarized proton beam from the ANL ZGS was focused by a quadrupole doublet onto the ANL-Michigan Polarized Proton Target (PPT-V). The beam was polarized in the vertical direction and the magnitude of the polarization averaged about $70 \%$ over the entire run. The target material consisted of propanediol beads doped with chromium paramagnetic complexes and contained in a resonant microwave cavity $4.1 \mathrm{~cm}$ long by $2.9 \mathrm{~cm}$ in diameter. The target cavity was situated in a magnetic ficld of $25 \mathrm{kG}$ and was maintained at a temperature of $0.5 \mathrm{~K}$. A $70 \mathrm{GHz}$ microwave system induced dynamic polarization in the free hydrogen protons in the propanediol. The direction of polarization was again vertical and its magnitude averaged about $70 \%$ over the ruIl. 


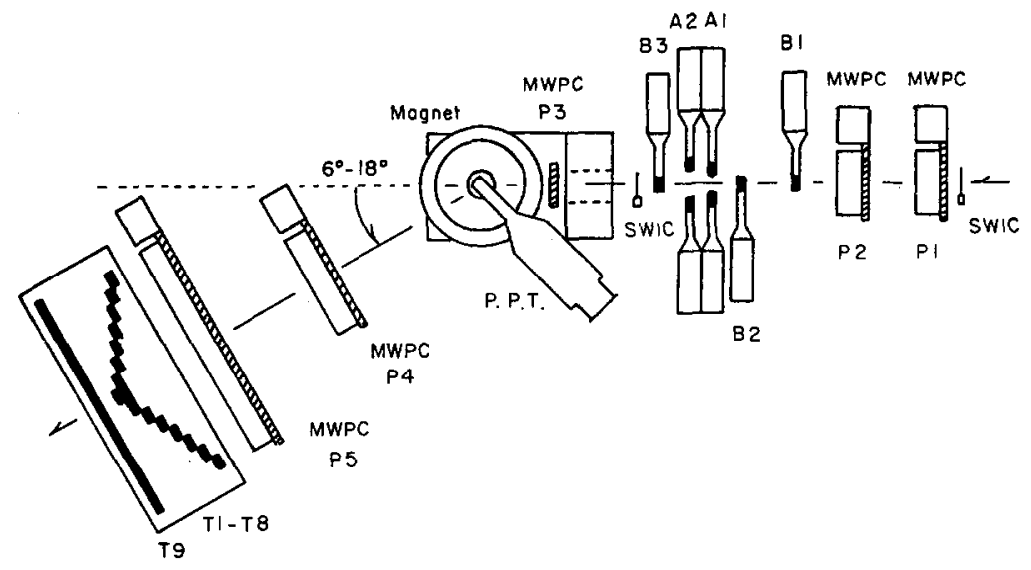

Fig. 1. Layout of the experiment showing beam and transmission counters (B and T), veto counters (A), multiwire proportional chambers (MWPC), and the polarized proton target (PPT).

The incident beam was defined by the scintillation counters B1, B2, and B3, and its halo vetoed by the hole counters $A 1$ and $A 2$. Unscattered particles and those scattered through small angles were detected by the transmission counters T1-T8. The large counter T9 was used in the event trigger and to monitor the efficiency of T1-T8.

Five multiwire proportional chambers (MWPC), P1-P5, each having two coordinate planes, were used for on-line reconstruction of the scattered and incident rays to ensure a proper focus of the beam within the polarized target. The downstream counters and MWPC's were mounted on a moveable arm which, pivoted under the center of the target, allowed easy repositioning of the system to compensate for beam deflection in the PPT magnetic field for each incident momentum.

All scintillator and MWPC signals were processed by the Rice data handling system [9] and written onto magnetic tape. All of the scintillator data and about $10 \%$ of the MWPC data were analyzed and displayed on line. The sign of the beam polarization was reversed on alternate ZGS pulses to average away the effect of any long term drifts in the beam position, spot size, intensity or spill structure. Other spurious asymmetries were checked by periodically reversing the direction of the target polarization and making use of the relations derived from rotational invariance,

$\sigma_{\mathrm{T}}(\uparrow \uparrow)=\sigma_{\mathrm{T}}(\downarrow \downarrow), \quad \sigma_{\mathrm{T}}(\uparrow \downarrow)=\sigma_{\mathrm{T}}(\downarrow \uparrow)$
In addition, test runs were taken with the target unpolarized and with the target cavity filled with nonhydrogenous material. $\Delta \sigma_{\mathrm{T}}$ as determined from these test runs was zero within statistical errors.

Data were taken in runs of about 30 minutes duration and the polarized target was reversed every 5 or 6 runs. Runs in which the beam was erratic in either steering or intensity and runs in which the shapes of the transmission distributions across the $T$ counters varied drastically for beam spin up and beam spin down were discarded.

The cross section difference for the $i$ th transmission counter is given by

$\Delta \sigma_{i}=\left(1 / N_{0} \rho t P_{\mathrm{B}} P_{\mathrm{T}}\right) \ln \left[T_{i}(\uparrow \uparrow) / T_{i}(\uparrow \downarrow)\right]$, where $N_{0}$ is Avogadro's number, $\rho=0.073 \pm 0.005 \mathrm{~g} / \mathrm{cm}^{3}$ is the density of free hydrogen protons in the PPT, $t=4.1 \mathrm{~cm}$ is the length of the PPT, $P_{\mathrm{B}}$ and $P_{\mathrm{T}}$ are the beam and target polarizations, and the $T_{i}$ 's are the ratio of the transmitted event coincidences, $\mathrm{B}_{1} \cdot \mathrm{B}_{2} \cdot \mathrm{B}_{3} \cdot \overline{\mathrm{A}}_{1} \cdot \overline{\mathrm{A}}_{2} \cdot T_{i}$, to the number of incident protons, $\mathrm{B}_{1} \cdot \mathrm{B}_{2} \cdot \mathrm{B}_{3} \cdot \overline{\mathrm{A}}_{1} \cdot \overline{\mathrm{A}}_{2}$, for beam and target polarizations parallel and antiparallel. $\Delta \sigma_{\mathrm{T}}$ was then obtained by extrapolating the $\Delta \sigma_{i}$ to zero solid angle. This extrapolation had only a few percent effect.

The measured values of $\Delta \sigma_{\mathrm{T}}$ are given in table 1 and shown in fig. 2 along with the previous measurements of deBoer et al. [8]. The errors shown for both sets of measurements are statistical errors only. An overall systematic error of the order of $10 \%$, due mainly to 
Table 1

Measured values of $\Delta \sigma_{\mathrm{T}}$.

\begin{tabular}{ll}
\hline$p(\mathrm{GeV} / c)$ & $\Delta \sigma_{\mathrm{T}}(\mathrm{mb})$ \\
\hline 1.2 & $\left.4.38 \pm 0.27^{\mathrm{a}}\right)$ \\
1.5 & $\left.3.00 \pm 0.26^{\mathrm{a}}\right)$ \\
1.75 & $\left.4.55 \pm 0.25^{\mathrm{a}}\right)$ \\
2.0 & $6.21 \pm 0.15^{\mathrm{a})}$ \\
& $\left.5.79 \pm 0.93^{\mathrm{b}}\right)$ \\
2.5 & $\left.2.20 \pm 0.31^{\mathrm{a}}\right)$ \\
3.0 & $0.76 \pm 0.20^{\mathrm{b})}$ \\
6.0 & $0.72 \pm 0.36^{\mathrm{b})}$ \\
6.0 & $0.34 \pm 0.07^{\mathrm{b})}$ \\
\hline
\end{tabular}

a) This experiment. b) DeBoer et al. [8].

uncertainty in the absolute values of the beam and target polarizations, was not included, to avoid obscuring structure in the data. The agreement of these results with those of deBoer et al. at $2.0 \mathrm{GeV} / c$ is quite good.

$\Delta \sigma_{\mathrm{T}}$ shows rather striking energy dependence. There is a broad peak at $2.0 \mathrm{GeV} / c$ followed by a smooth decrease with increasing energy. This decrease is possibly due to the opening of more and more inelastic channels with different spin dependences which tend to cancel one another [4]. The energy dependence below 2.0 $\mathrm{GeV} / c$ is not easily understandable. There is no obvious correlation between this structure and the opening of inelastic channels, i.e. pp $\rightarrow$ pn $\pi^{+}$at $0.8 \mathrm{GeV} / c, \mathrm{pp} \rightarrow$ $\mathrm{n} \Delta$ at $1.3 \mathrm{GeV} / c$ and $\mathrm{pp} \rightarrow \Delta \Delta$ at $2.1 \mathrm{GeV} / c$. The curves shown in fig. 2 are a prediction of simple exchange models for the contribution of one of these channels, $\mathrm{pp} \rightarrow \mathrm{pn} \pi^{+}[10]$.

The major contribution to the total cross section in this region is still the elastic cross section which has a broad maximum $(30 \mathrm{mb})$ at $1.5 \mathrm{GeV} / c[11]^{\neq 1}$. The contribution of elastic scattering to $\Delta \sigma_{\mathrm{T}}$ is given by ${ }^{+2}$

$\Delta \sigma_{\mathrm{T}}^{\mathrm{el}}=\int\left(C_{\mathrm{nn}}+C_{\mathrm{ss}}\right) \frac{\mathrm{d} \sigma}{\mathrm{d} \Omega} \mathrm{d} \Omega$

Unfortunately, the data on the spin correlation parameters, $C_{\mathrm{nn}}$ and $C_{\mathrm{ss}}$, are poor or non-existent in this

₹1 A more recent compilation has also been made by A. Yokosawa.

\$2 For definitions of these parameters, see, for example, ref. [12].The indices nn and ss refer to spins perpendicular to and lying in the scattering plane.

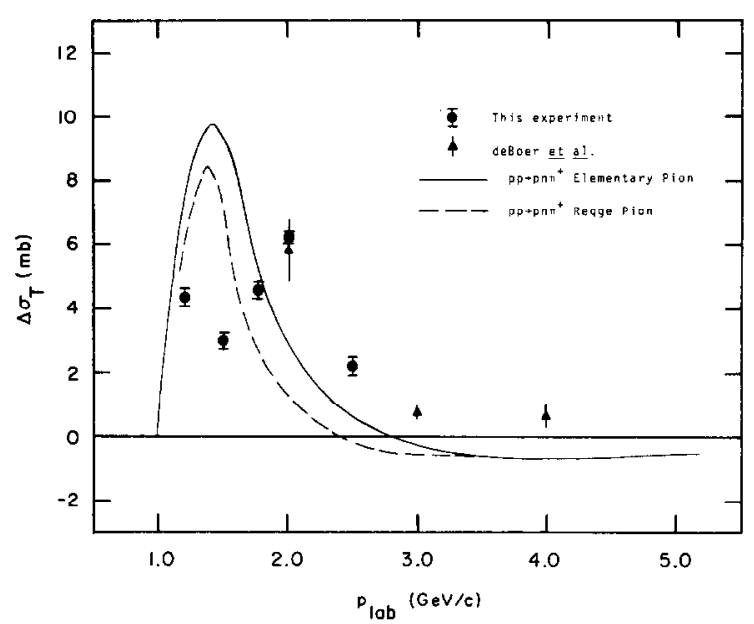

Fig. 2. Measured values of $\Delta \sigma_{\mathrm{T}}$. The curves show the predictions of simple exchange models for the contribution of the channel $\mathrm{pp} \rightarrow \mathrm{pn} \pi^{+}$.

energy range. There is a clear need for good measurements of these parameters at these energies. This would allow a measurement of the contribution of elastic scattering to $\Delta \sigma_{\mathrm{T}}$, and thus the separation of its elastic and inelastic parts.

Auer et al. [13] have measured the longitudinal spin dependence of the total cross section, $\Delta \sigma_{\mathrm{L}}$, over the same momentum range as this experiment. It is interesting to note that their results also show structure in the vicinity of $1.5 \mathrm{GeV} / c$. They interpret this structure [14], in conjunction with certain phase shift analyses [15-17] and pp elastic polarization mcasurements [18-20], as evidence for the existence of a diproton resonance with a mass around $2000 \mathrm{MeV} / \mathrm{c}^{2}$. The structure in $\Delta \sigma_{\mathrm{T}}$ may be further evidence for phenomena of this nature.

We would like to thank the entire ZGS staff for their efforts in providing the polarized beam and Mr. J.A. Bywater for his assistance in running PPT V. We also thank Messrs. M. Calkin, K. Johns, and R. Pool for their assistance in the analysis of the data. Three of us, K. Abe, R.C. Fernow and T.A. Mulera, thank Professor A.D. Krisch for support during the running of this experiment. 


\section{References}

[1] M. Jacob and G.C. Wick, Ann. Phys. 7 (1959) 404.

[2] S. Mandelstam, Proc. Roy. Soc. A244 (1958) 491.

[3] G.L. Kane and G.H. Thomas, Argonne Report, ANLHEP-PR-75-56 (1975).

[4] E.L. Berger, P. Pirila and G.H. Thomas, Argonne Report, ANL-HEP-PR-75-72 (1975).

[5] G.N. Epstein and D.O. Riska, Michigan State Preprint (1976) unpublished.

[6] W.M. Kloet, R.R. Silbar, R. Aaron and R.D. Amado, LASL Report LA-UR-77-2321 (1977); and to be published.

[7] E.F. Parker et al., Phys. Rev. Lett. 31 (1973) 783.

[8] W. deBoer et al., Phys. Rev. Lett. 34 (1975) 558.
[9] J.A. Buchanan et al., Nucl. Instr. Meth. 99 (1972) 159; IEEE Trans. Nucl. Sci. NS-20 (1973) 691.

[10] E.L. Berger, Argonne Report, ANL-HEP-PR-76-35 (1976).

[11] E. Bracci et al., CERN Report, CERN/HERA 73-1.

[12] M.J. Moravcsik, The Two Nucleon Interaction (Clarendon, Oxford, 1963).

[13] I.P. Auer et al., Phys. Lett. 67B (1977) 113; 70B (1977) 475.

[14] H. Hidaka et al., Phys. Lett. 70B (1977) 479.

[15] W. Grein and P. Kroll, Wuppertal Report No. WU B 77-6 (1977).

[16] M.H. MacGregor et al., Phys. Rev. 169 (1968) 1149.

[17] N. Hoshizaki, Progr. Theoret. Phys. 57 (1977) 1099; N. Hoshizaki and T. Kadota, Progr. Theoret. Phys. 57 (1977) 335 .

[18] A. Yokosawa et al., Phys. Rev. Lett. 16 (1966) 714.

[19] B.A. Ryan et al., Phys. Rev. D3 (1971) 1.

[20] M.G. Albrow et al., Nucl. Phys. B23 (1970) 445. 